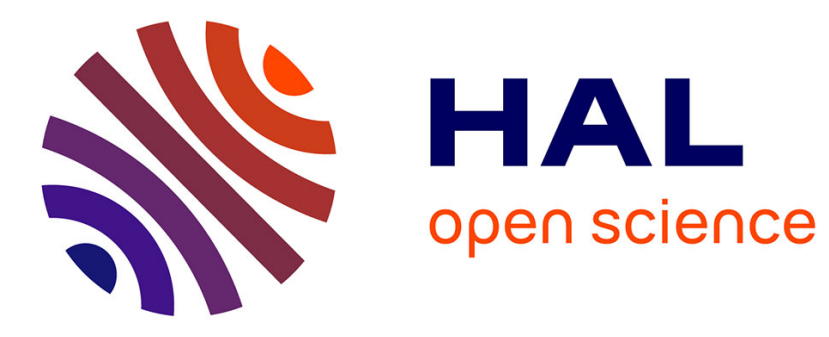

\title{
MINIATOM: Miniaturized Coherent Atom Sensors
}

\author{
Baptiste Battelier
}

\section{To cite this version:}

Baptiste Battelier. MINIATOM: Miniaturized Coherent Atom Sensors. Conference on Lasers and Electro-Optics/International Quantum Electronics Conference, May 2009, Baltimore, United States. pp.JThE101. hal-00630114

\section{HAL Id: hal-00630114 \\ https://hal-iogs.archives-ouvertes.fr/hal-00630114}

Submitted on 12 Oct 2011

HAL is a multi-disciplinary open access archive for the deposit and dissemination of scientific research documents, whether they are published or not. The documents may come from teaching and research institutions in France or abroad, or from public or private research centers.
L'archive ouverte pluridisciplinaire $\mathbf{H A L}$, est destinée au dépôt et à la diffusion de documents scientifiques de niveau recherche, publiés ou non, émanant des établissements d'enseignement et de recherche français ou étrangers, des laboratoires publics ou privés. 


\title{
MINIATOM: Miniaturized Coherent Atom Sensors
}

\author{
Author: BATTELIER Baptiste \\ Institut d'Optique Graduate School \\ Campus Polytechnique RD 128 \\ 91127 Palaiseau Cedex FRANCE \\ email: baptiste.battelier@institutoptique.fr
}

\begin{abstract}
We conceive and build a compact cold atom interferometer with an original architecture based on integrated optical components. These new inertial sensors can play a significant role in navigation, fundamental physics and earth observation.
\end{abstract}

\section{Summary:}

Inertial sensors using atomic interferometry is a potential breakthrough technology for high precision accelerometers and gyrometers used in land, air or spatial navigation or geodesy. These high precision sensors offer also new developing prospects concerning Earth observation, fundamental physics, metrology and boreholes gravimeter. The exploiting of this cold atoms sensors is based on the possibility to miniaturize and simplify the current systems developed in the research groups. This miniaturization needs both fundamental investigation on new atomic sources and efforts to turn this laboratory devices into compact, reliable and self-working systems. The main goal of this project is to conceive and build a compact cold atom interferometer. To reach this goal, this project gave birth to a collaboration between research laboratory (Institut d'Optique, Syrte) and manufacturers specialized in integrated optical sensors (THALES, IXSEA) and in integrated optics (KLOE).

Atomic interferometers have a strong potential for several applications:

- as a gyrometer for spatial and submarine applications. Currently, the best existing inertial sensors use two kinds of technologies: electromechanical systems and laser systems.

- as an accelerometer. Atomic devices have already reached much better performances than the existing solutions (cf figure 1).

- as a gradiometer. It is a promising application for navigation. The cold atoms allow to make several accelerometers with the same laser and atom sources, consequently the expected precision is better than those get by comparing independent devices as it is done with the current systems.

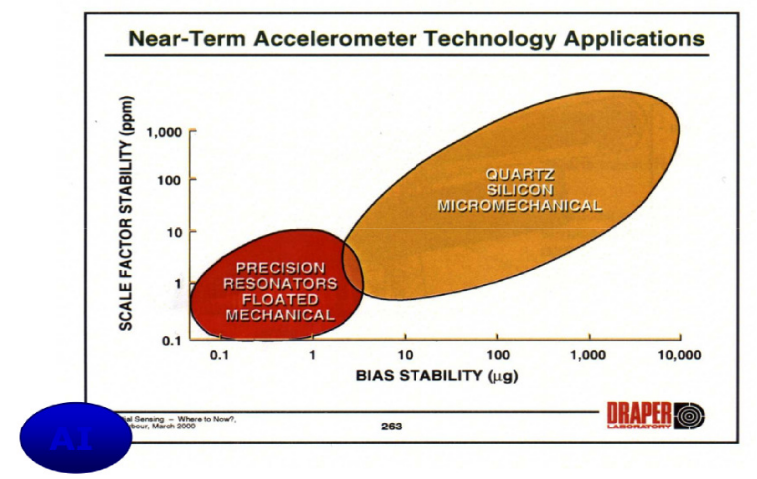

figure 1: Performances of the current accelerometers (red and orange) compared to cold atoms sensors (blue) [1].

The main goal of MINIATOM project is to overstep the performances of the current inertial sensors and to decrease the experimental complexity and the size of cold atoms systems. The next generation of cold atoms sensors must be compact, transportable, and self-working. These systems are naturally highly accurate, stable and reliable. The miniaturization of these devices must help to reduce cost in the future. Considering the high performances of this atomic detectors, a compromise between performances and operational longevity must allow a larger variety of applications. The miniaturization of these systems is based on a strong integration of devices widely used in cold atoms laboratory in general, in those working especially on atomic interferometry but also on topics as quantum information, degenerate gases, and precision measurement. All the research groups in those fields would enjoy the availability of a reliable source for atoms cooling. For this purpose, a effort will be made on hybridization technology of several integrated functions on the same ship.

The interferometer can be split into two parts: the laser source containing all the lasers and needed electronics components and the sensor architecture containing the atomic source and tools to control the atoms if necessary. 


\section{1) Laser system}

In this project, we focus on the use of integrated optical components. The main idea is to develop a structure based on efficient telecommunication components, available and perennial. Thanks to second harmonic generation, it is possible to obtain the wavelength corresponding to the atomic transition of Rubidium or/and Potassium. The ideal set of components, who would allow to get a miniature source is not available. Currently, some components (splitters, SHG crystals) doesn't fulfill needed specifications. First, we focus on a crossed strategy trying to combine all the advantages of these components. The laser source can be a fiber laser or an external cavity laser in order to have a tight linewidth $(10 \mathrm{kHz}$ or $50 \mathrm{kHz}$ ). The frequency lock part will be made with telecommunication technology and second harmonic generation. After frequency conversion, semiconductor tapered amplifier (TA) is used to reach power between $100 \mathrm{~mW}$ and $1 \mathrm{~W}$. Generally, cold atoms experiments need several beams: an integrated beamsplitter based on multimodal interferences (MMI) can be directly connected to the TA output. If you combine this MMI waveguide with an electrical controller, you can modulate the power of each output. The whole laser system is based on the use of fiber optics, and collimation devices to deliver all the beams around the vacuum system. The project benefit of the abilities of IXSEA in fiber optics which is a critical part of the connection between the laser system and the atomic device. The possibility to conceive polarizing fibers, maintaining the polarization with an extinction ratio better than $0,1 \%$ is planned.

The key component is the non linear PPLN waveguide for the second harmonic generation. This component is compatible with a "all-fibered" structure and provide a high conversion efficiency (until 2000\%/W). This efficiency is much higher than those with bulk crystals (a few \%/W). We can expect to have $50 \%$ efficiency if we work with enough input power. This would be an optimal solution for miniaturization and electrical consumption. Such a standard component doesn't currently exist for relatively high power, typically $100 \mathrm{~mW} @ 780 \mathrm{~nm}$. The power inside the crystal is limited by photorefractive effects. To avoid this problem we must have a high phase matching temperature (higher than $100^{\circ} \mathrm{C}$ ), which is not possible because of the pigtail. The French spatial agency CNES and KLOE decided to develop new solutions to improve the pigtail.

This new architecture of a laser source was validated in micro-gravity environment with a experience put on a plane who make parabolic flights. During the last campaign in October 2009, we succeed in producing Ramsey fringes. At our knowledge, it is first atomic clock with Raman transitions in microgravity. This new architecture led to submit a patent.

\section{2) New sensor architecture}

The inertial sensor architecture is usually linked to the configuration of the atomic source. In a classical configuration, 6 beams is needed for a 3D magneto optical trap. Moreover, 2 additional beams are needed for the Raman transitions of the interferometer. One main goal is to reduce the number of needed beams for the accelerometer. For example, a reflecting corner allow to use only one beam for the magneto optical trap (figure 2 and reference [2]). The sensor architecture also led to submit a patent (08/5/599).

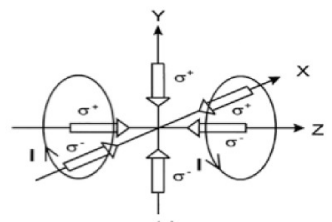

(a)

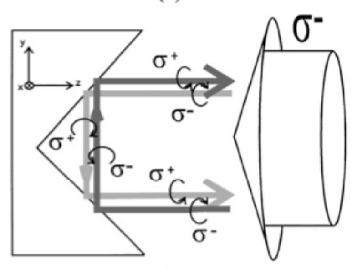

(b)

Figure 2: a classical magneto-optical trap needs six beams. With one reflecting corner, it is possible to have the six directions with only one beam [2]

Even though laser cooling is good enough for cold atoms accelerometers, the control of the source positioning can be a problem. Indeed, in a magneto-optical configuration, position precision and stability is linked to magnetic field and light intensity stability. The use of non dissipative trap, whose center is exactly defined by the minimum potential energy, optical or magnetic, seems to be a better option. For instance, it is possible to realize an optical trap with a telecommunication fiber amplifier. 